
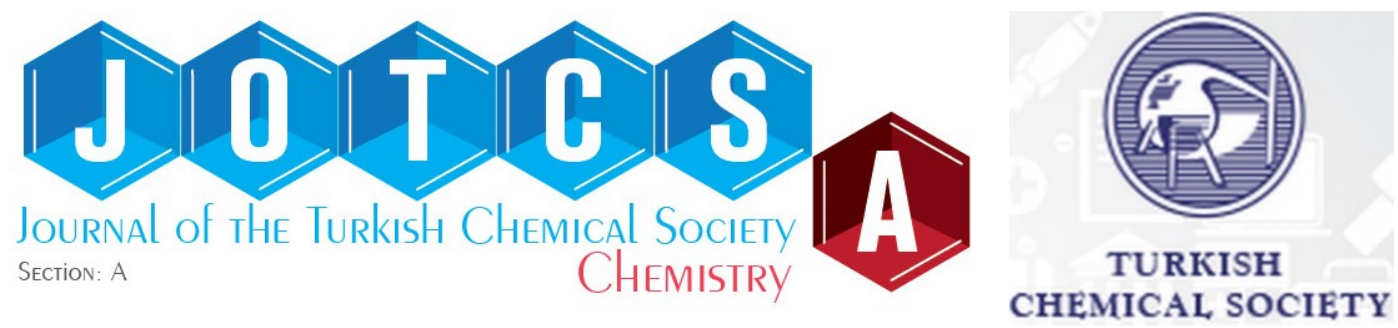

\title{
Fabrication and Characterization of Gelatin/Chitosan Hydrogel Membranes
}

\author{
Thi Sinh Vo' ${ }^{1 *}$, Tran Thi Bich Chau Vo ${ }^{2} D$, Truong Sinh Nguyen ${ }^{1}(\mathbb{D}$, and \\ Tran Trung Tien ${ }^{1}$ \\ ${ }^{1}$ School of Mechanical Engineering, Sungkyunkwan University, Suwon 16419, Korea.
2Department of Industrial Management, Can Tho University, Can Tho, Vietnam.
}

\begin{abstract}
The gelatin/chitosan (GEL/CTS, GC) membranes have been fabricated well by a simple in-situ method. The as-prepared GC membranes were characterized by morphological surface (scanning electron microscopy, SEM), chemical (Fourier-transform infrared spectroscopy, FT-IR), crystallinity (X-ray diffraction, XRD), thermal (thermal gravimetric analysis, TGA), mechanical (tensile test), hydrophilic (water contact angle), and swelling properties to elucidate the changes in their chemical structures and morphologies. The morphological structure of the GC membranes was found to be very smooth, non-rough and homogeneous. The FT-IR and XRD studies manifest that the GC membranes have good interaction and compatibility between GEL and CTS in the hydrogel mixture. The prepared GC membranes also obtain better thermal, mechanical and swelling properties comparing to the raw CTS molecule. These results suggest that the nontoxic GC membrane can become a preferred hydrogel membrane in the field of wound dressing or tissue-engineering applications.
\end{abstract}

Keywords: Chitosan, gelatin, swelling, hydrogel membrane.

Submitted: May 25, 2021 . Accepted: September 20, 2021.

Cite this: Vo TS, Vo TTBC, Nguyen TS, Tien TT. Fabrication and Characterization of Gelatin/Chitosan Hydrogel Membranes. JOTCSA. 2021;8(4):1045-56.

DOI: https://doi.org/10.18596/jotcsa.942478.

*Corresponding author. E-mail: vtsinh92@skku.edu.

\section{INTRODUCTION}

As known, polymeric membrane system was extensively applied in different research fields and practical applications (1-5), at the same time that remarkable factors of a polymeric membrane almost regard to possibly physical, chemical and interface properties. Hence, they are necessary to conduct considerable modification methods that can obtain better desired results corresponding to multiple researches. Especially, hydrogel membrane-based superabsorbent has effective swelling behaviors that can be due to the hydrophilic functional groups (i.e., carboxyl, amino, and hydroxyl groups) contained availably on the polymer chains (6-14). At the same time, the hydrogel membranes are also considered as hydrophilic membranes with three-dimensional cross-linked networks being applied for plenty of biomedical applications. These hydrogel membranes significantly swell in an aqueous medium that can be mainly due to their cross-linked networks, meaning they do not disintegrate in water at physiological pH and temperature (15). Furthermore, the hydrogel membranes reach lots of advantages, e.g., high water content and soft elastic property, boost granulation and epithelialization due to the moist environment (16), promote the healed wound without any damage (17), respectively. Thus, the hydrogel membrane is widely used in the fields of drug delivery, agriculture, purification, hygiene, and food industry. The development and expansion of eco-friendly membrane has been researched and explored intensively to protect environment; concomitantly, multiple natural sources are recently concerned (10-14). 
Notably, single-network hydrogel membranes show slow response and weak mechanical property at swelling, while multiple-network membranes (i.e., interpenetrating polymer networks) can be designed to overcome this drawback (18). So far, plenty of hydrophilic polymer sources have been used to produce the appropriate hydrogel membranes, counting natural (proteins and polysaccharides) and synthetic (contain hydrophilic functional groups, i.e., carboxyl, amino, and hydroxyl groups, etc.) polymer sources; especially for commonly natural polymers, e.g., gelatin and chitosan. Specifically, chitosan (CTS) is considered to be an eco-friendly and widely-used compound of chitin $\mathrm{N}$-deacetylation, which is also a potential candidate in numerous fields such as drugs, catalysis, food, etc. $(1,2,6-8)$. Besides, the CTS is known as a biomedical material source owing to its wound-healing ability, biodegradability, biocompatibility, hemostasis, antimicrobial activity, etc. (19-21), which leads to being a valuable biomedical material - CTS molecule. Concomitantly, the CTS is soluble in weak acid solvents (i.e., lactic acid, acetic acid, etc.) but insoluble in a water medium, although it has a crystalline structure with several hydrogen bonds (22). Indeed, several researchers presented possible modifications on basic of the CTS molecule regarding to available chemical features to be expanded in the material systems of beads, fibers and membranes, owing to its good solubility in a weak acid medium $(1,2,23-27)$. By the way, gelatin (GEL) is a biocompatible protein with a very high bio-absorptivity and low antigenicity in a living body $(28,29)$, and its outstanding property regards to a sol-gel transition under an aqueous medium. Several various membranes have been produced on natural material sources of the CTS and GEL molecules to be applied in biomedical applications $(2,26,28)$. Nonetheless, these membranes were mostly successfully prepared by casting method using GEL/CTS (GC) solution in acetic acid solvent, which is known as a common solvent to dissolve the CTS molecules. However, the odor feature of this acid solvent is one of the disadvantages to be utilized in the fabrication of GC membranes. Hence, the lactic acid solvent is selected to replace the acetic acid solvent and overcome this disadvantage in this study, at same time that the incorporation of GEL and CTS molecules is also considered as a nontoxic hydrogel membrane, owing to natural polymer sources.

Herein, the lactic acid solvent is employed to fabricate GC hydrogel membranes based on a simple in-situ method. Moreover, the morphological surface, crystallinity, thermal, mechanical, hydrophilic, and swelling properties of the asprepared GC membranes are specifically investigated with different weight ratios of GEL/CTS to probably elucidate the changes in their chemical structures and morphologies. Thereby, we hope that these nontoxic GC membranes can become a promising hydrogel membrane in the fields of wound dressing or tissue-engineering applications.

\section{EXPERIMENTAL SECTION}

\section{Materials}

CTS (deacetylation degree $=\sim 90 \%$ ) and lactic acid $\left(\mathrm{CH}_{3} \mathrm{CH}(\mathrm{OH}) \mathrm{COOH}, 85-90 \%\right)$ are obtained from Sigma Aldrich. GEL is purchased from Samchun Chemical Co.. Deionized water is directly offered from a Milli-Q ultrapure system.

\section{Fabrication of GC Membranes}

A CTS stock solution with a concentration of $2 \%$ is fabricated by mixing CTS in $100 \mathrm{~mL}$ of aqueous lactic acid $(2.5 \%, \mathrm{v} / \mathrm{v})$ and stirring overnight. Then GEL is added and stirred in $20 \mathrm{~mL}$ of distilled water at $50{ }^{\circ} \mathrm{C}$, mixed into the CTS solution, and agitated at $50{ }^{\circ} \mathrm{C}$ with the different weight ratios (GEL:CTS, wt/wt $=8: 2 ; 6: 4 ; 5: 5 ; 4: 6$ and $2: 8$ ). The GC solution with different weight ratios is spread over the bottom of Petri dishes (100 mm diameter), and resultant GC membranes are air-dried for 1 day (room temperature). The fabrication process of the GC hydrogel membrane is described in Figure 1. 


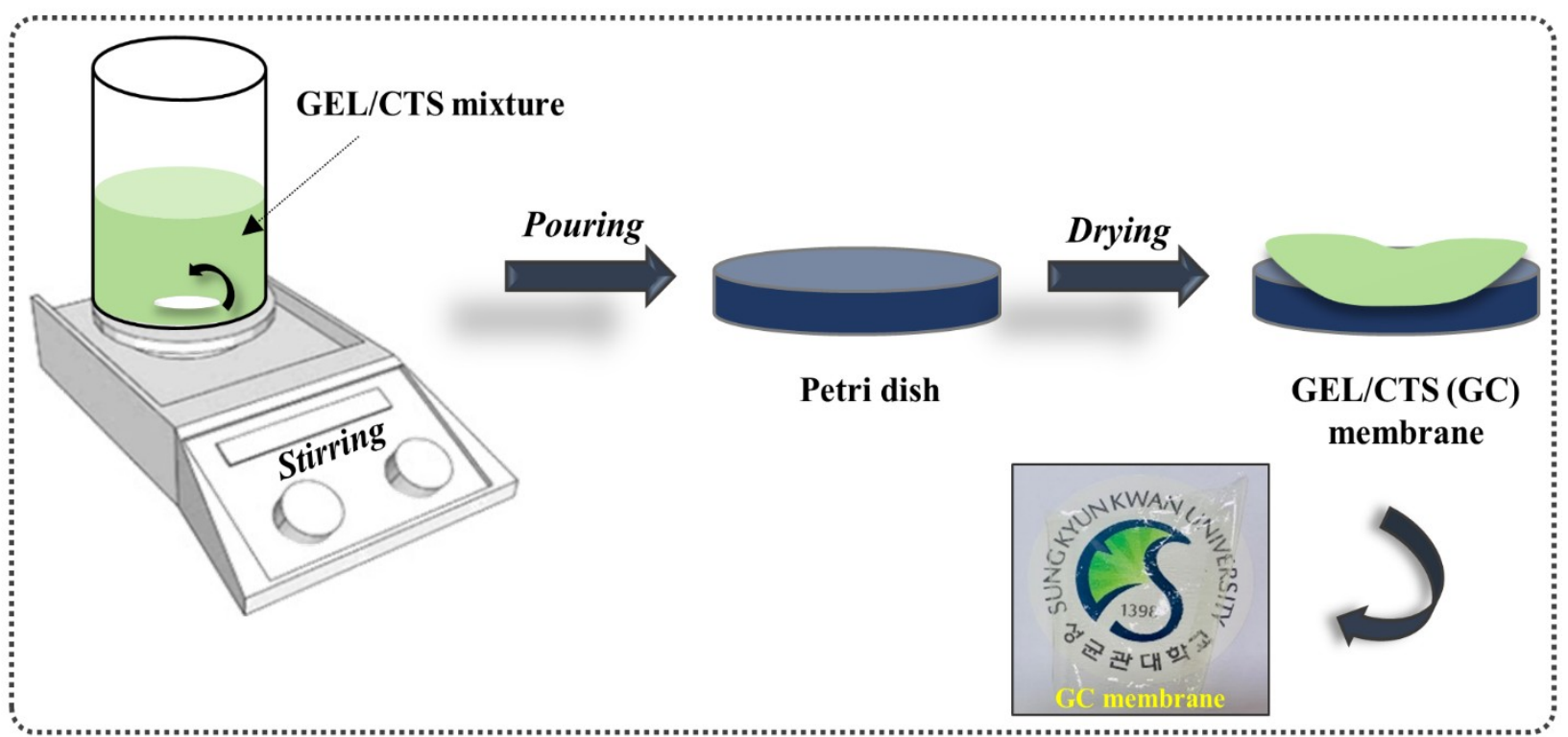

Figure 1: Fabrication process of GC hydrogel membrane.

\section{Swelling Study}

The swelling property of the prepared GC membranes is performed by immersing and sealing into an aqueous solution. Specifically, the asprepared GC membranes are cut and measured the weight $\left(2 \mathrm{~cm} \times 2 \mathrm{~cm}, \mathrm{~W}_{0}\right.$ ) by a four-digit analytical balance. Next, the cut membranes are immersed and sealed into distilled water at room temperature. After preset times ( $t=5-60 \mathrm{~min}$ ), the membranes are took-out and weight was measured $\left(\mathrm{W}_{1}\right)$. Finally, the swelling rate (SW, \%) of the membrane is calculated from an equation: SW $(\%)=\left[\left(W_{1}-\right.\right.$ $\left.\left.\mathrm{W}_{\mathrm{o}}\right) / \mathrm{W}_{\mathrm{o}}\right] \times 100$.

\section{SEM Analysis}

The morphological structures of the GC membranes (i.e., top-surface and cross-section) are captured from scanning electron microscopy (SEM) (FESEM JSM-7600F) at different magnifications.

\section{FT-IR and XRD Analysis}

The chemical and crystalline characterization of the GC membranes are analyzed by a Fourier-transform infrared (FT-IR) spectrometer and an X-ray diffraction (XRD). FT-IR spectroscopy is measured in the $4000-500 \mathrm{~cm}^{-1}$ wavenumber region by an FT-IR spectrometer with the $\mathrm{KBr}$ method (Nicolet 380 spectrometer). Wide-angle XRD analysis is performed in the $2 \theta$ range of $5-70^{\circ}$ via an $X$-ray diffractometer (D8 ADVANCE, Bruker Corporation).

\section{Thermal Gravimetric Analysis}

Thermal gravimetric analysis (TGA) is conducted on a Seiko Exstar6000 instrument with a temperature range of $30-700^{\circ} \mathrm{C}$ at a heating rate of $10^{\circ} \mathrm{C} \cdot \mathrm{min}^{-1}$ for the samples.

\section{Contact Angle Measurement}

In order to determine the hydrophilic properties of the GC membranes, a drop technique is applied to investigate the water contact angle [SEO Phoenix $\mathrm{MT}(\mathrm{M})$ ] by using a distilled water drop (volume $=5$ $\mu \mathrm{L}$ ). The contact angle values are calculated by analyzing the captured drop images based on the

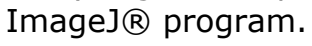

\section{Tensile Analysis}

The strength and strain of the tensile test (original size of membrane $=1 \mathrm{~cm} \times 5 \mathrm{~cm}$ ) are carried out by a universal tensile machine (UTM model 5565, UK) with $250 \mathrm{~N}$ of load cell and $10 \mathrm{~mm} \cdot \mathrm{min}^{-1}$ of pulling rate. Prior to the tensile test, the membranes are stored for more than one day at room temperature.

\section{RESULTS AND DISCUSSION}

\section{Characterization of GC Membranes}

As known, several researchers (30-32) have used acetic acid solvent, which is known as a common solvent to dissolve the CTS molecules; however, the odor nature of this solvent is seen as a disadvantage to be utilized in the fabrication of GC membrane. Thus, the lactic acid solvent is chosen to replace acetic acid in this study as well as to overcome this disadvantage. 
(a)

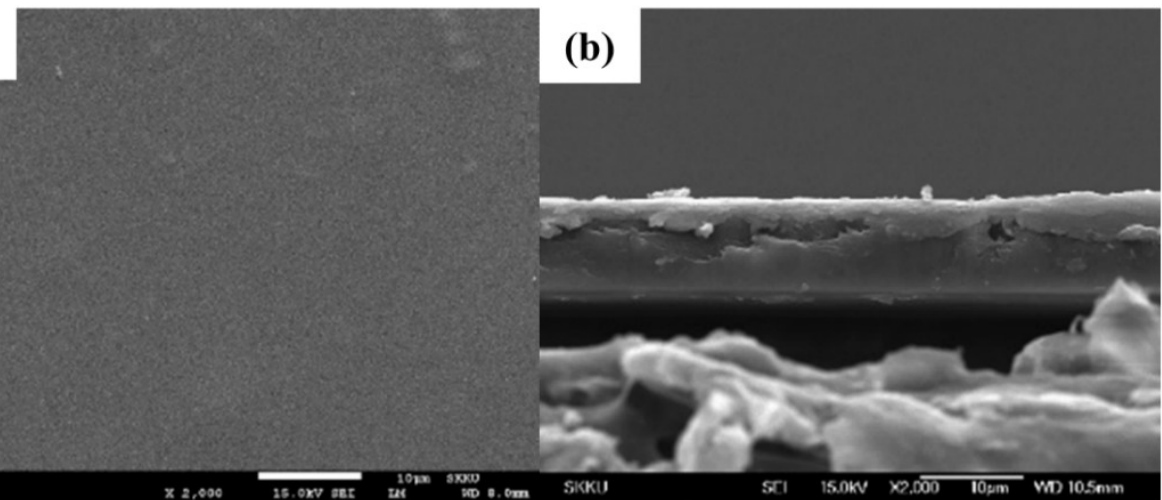

Figure 2: SEM images of top-surface (a) and cross-section (b) of GC_5/5 membrane.

For a hydrogel membrane, the morphological structure is also concerned to be one of the significant factors to evaluate its performance corresponding to the aim study (2). In this study, the morphological structure of GC membranes is detected by SEM images (Figure 2), indicating that the top-surface (Figure 2a) and cross-section (Figure 2b) morphologies of the GC_5/5 membrane are relative smooth surface and non-rough without the pores. Thereby, this smooth morphology is owing to the GC hydrogel formation in the membrane, manifesting that the CTS and GEL molecules are well dispersed in the molecular level, as well as the homogeneous characterization of this GC mixture. As such, the smooth surface and homogeneous feature of the GC hydrogel membrane are truly useful in the field of wound dressing or tissue engineering applications.

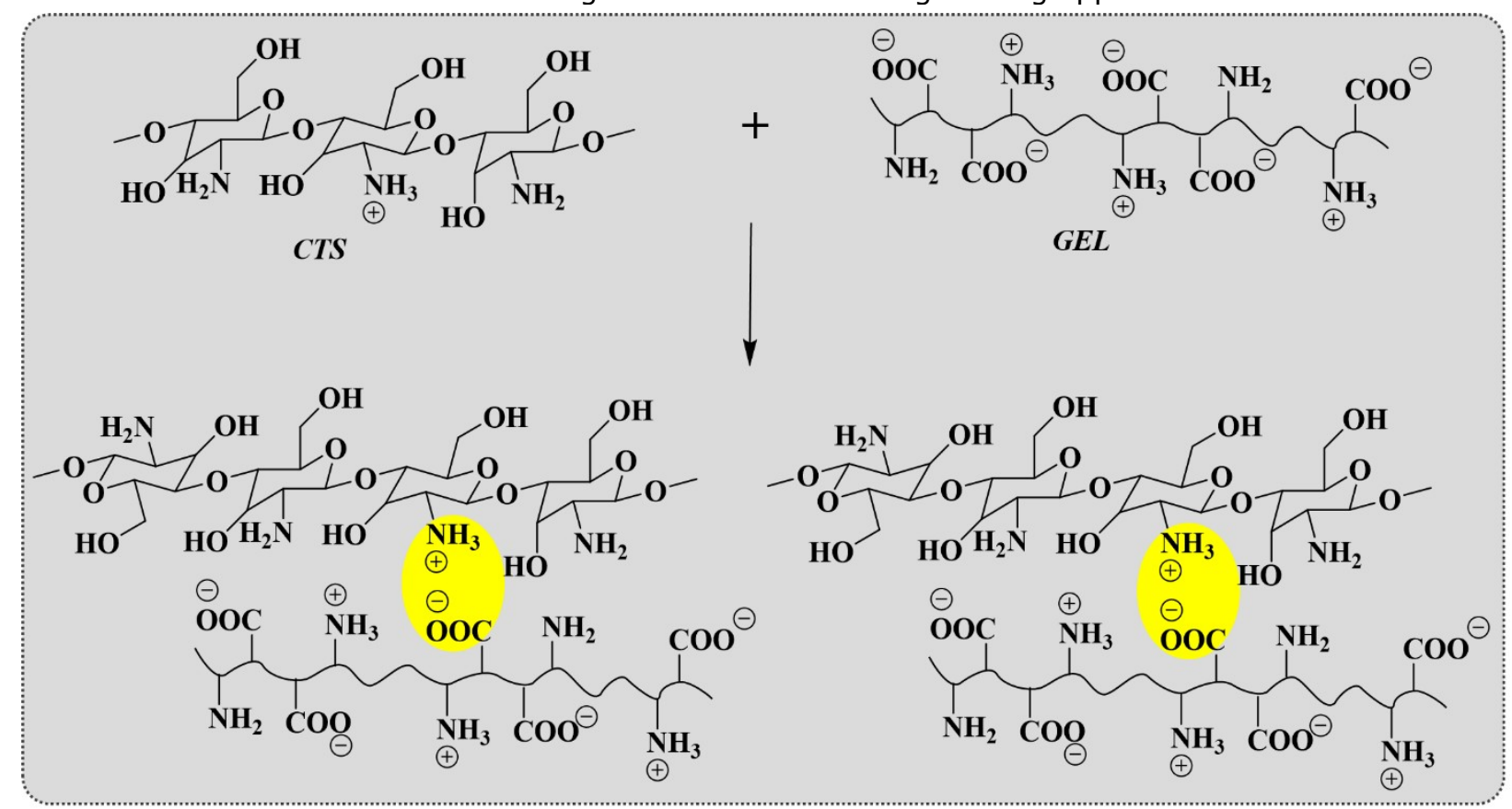

Figure 3: Ion exchange in lactic acid solvent and possible reaction mechanism between CTS and GEL.

Figure 3 indicates that in the GEL/CTS spectrum, the $\mathrm{C}=\mathrm{O}$ groups of $\mathrm{GEL}$ absorbed with the $\mathrm{N}-\mathrm{H}$ groups of CTS resulting in strong hydrogel bonds, leading to a far more miscible GEL/CTS component. To further understand the chemical characterization of the membrane, FT-IR spectra of CTS, GEL, and GC membrane are shown in Figure $4 a$. The peaks at $3500-3000 \mathrm{~cm}^{-1}$ can belong to the $\mathrm{O}-\mathrm{H}$ and $\mathrm{N}-\mathrm{H}$ stretching of CTS, and the peak at $1560 \mathrm{~cm}^{-1}$ regards to the $\mathrm{C}-\mathrm{N}$ deformation vibration (amide $\mathrm{II}$ ) of CTS $(33,34)$. The peaks at the amide $\mathrm{I}(\mathrm{C}=0)$ band $\left(1645 \mathrm{~cm}^{-1}\right)$ and the amide II band are very weak that can be due to the higher deacetylation degree of CTS $(33,34)$. For GEL spectra, the $\mathrm{N}-\mathrm{H}$ bending vibrations of the amide I, II, and III bands can be observed at $1655 \mathrm{~cm}^{-1}, 1510 \mathrm{~cm}^{-1}$, and 1324 $\mathrm{cm}^{-1}$, respectively (35). Moreover, the vibrational peak of the GC membrane relating to amino groups at $1560 \mathrm{~cm}^{-1}$ has disappeared after GEL/CTS polymerization, while prominent peaks at $1650 \mathrm{~cm}^{-1}$ are observed clearly in the GC membrane spectra. Thereby, these are probably explained as based on the amidation reaction between carboxyl and amino occurring in CTS and GEL mixture (Figure 3). 
Besides, the peaks at $3500-3000 \mathrm{~cm}^{-1}$ have also became broader in the GC membrane spectra regarding their hydrogen bonding interaction occurred in that GC hydrogel mixture. At the same time, the shifted peaks of $\mathrm{C}=\mathrm{O}$ and $\mathrm{C}-\mathrm{O}-\mathrm{C}$ are observed from $1645 \mathrm{~cm}^{-1}$ to $1539 \mathrm{~cm}^{-1}$, and from $1068 \mathrm{~cm}^{-1}$ to $1058 \mathrm{~cm}^{-1}$ in the GC membrane spectra, respectively. Thereby, the chemical interactions of GEL and CTS molecules have happened in this hydrogel mixture, similarly to the above-mentioned SEM analysis. In addition, Figure $4 \mathrm{~b}$ manifests the XRD pattern of the CTS, GEL, and GC membrane. These show that the primary

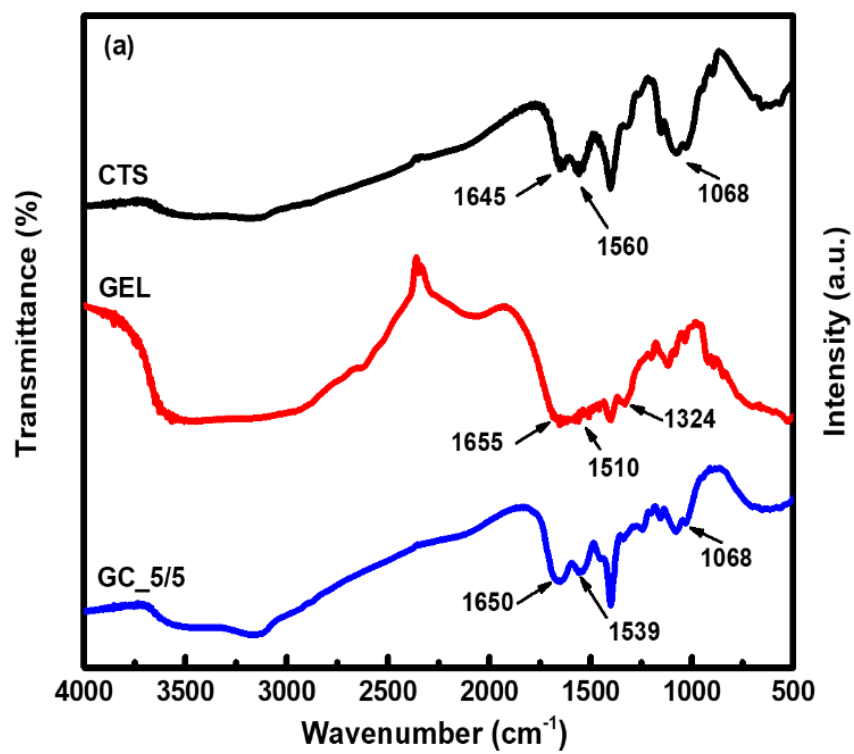

diffraction peaks are observed at $\sim 20^{\circ}$ in their XRD patterns, suggesting good interaction and compatibility between GEL and CTS in the hydrogel membrane. Concomitantly, the peak intensity ratio of GC membrane is reduced with a supplement of GEL component into the mixture, resulting in a decrease in CTS crystallinity probably because of the incorporation of the amorphous nature of GEL into this mixture. Besides, a small reduction in the GC membrane crystallinity can be due to the hydrogen bonding interactions between the GEL and CTS molecules in the mixture regarding their good compatibility $(36,37)$.

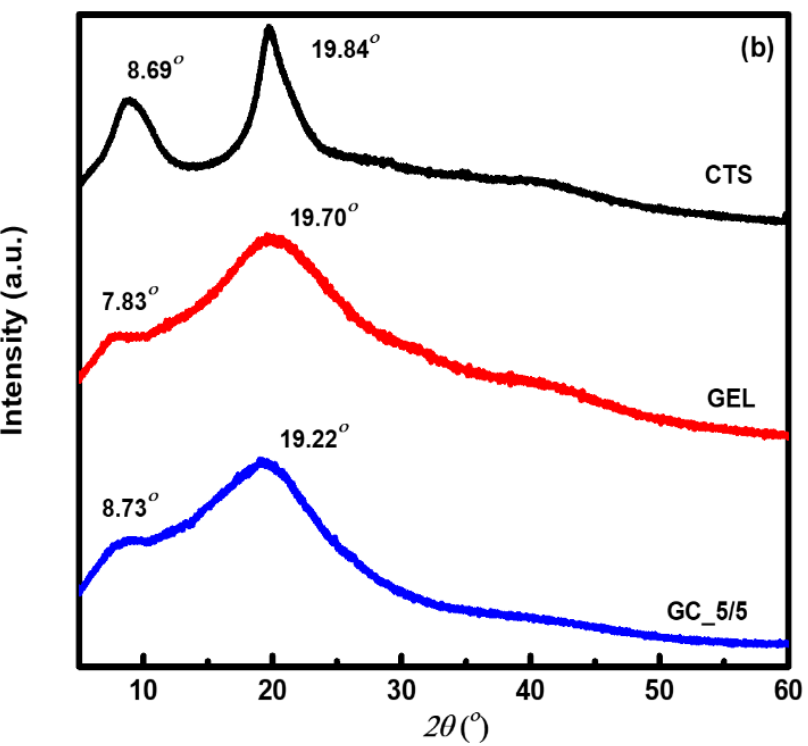

Figure 4: FTIR (a) and XRD (b) spectra of CTS, GEL, and GC_5/5 membrane.

Furthermore, the thermal property of a material is also concerned as a significant factor to be investigated in this study. In Figure 5, it illustrates the thermograms of CTS, GEL, and GC membrane. Their loss of initial weight at $\sim 50{ }^{\circ} \mathrm{C}$ is attributed to their loss of moisture in all samples. In addition, the CTS curve shows lower thermal stability than that of the GC membrane. Specifically, the second degradation of the CTS curve is observed at $295^{\circ} \mathrm{C}$, while that of the GC membrane is at $319^{\circ} \mathrm{C}$. Thereby, it probably relates to incorporating the amorphous nature of GEL into the mixture, as well as the GEL degrades faster than the GC membranes. Besides, the DTG peaks of the GC membrane are similar to CTS peaks, while those of GEL are not similar to the GC membrane (Figure $5 \mathrm{~b}$ ) that can be due to the difference in crystal structure and hydrogen bonding network between GEL and CTS. Also, it indicates that the as-prepared membranes with CTS and GEL are well dispersed at the molecular level. As a result, combining these materials to form a hydrogel membrane is stable more in thermal property compared with pure materials. Overall, there are good interactions and compatibility between the GEL and CTS molecules in this hydrogel mixture through the aforementioned analyses. 

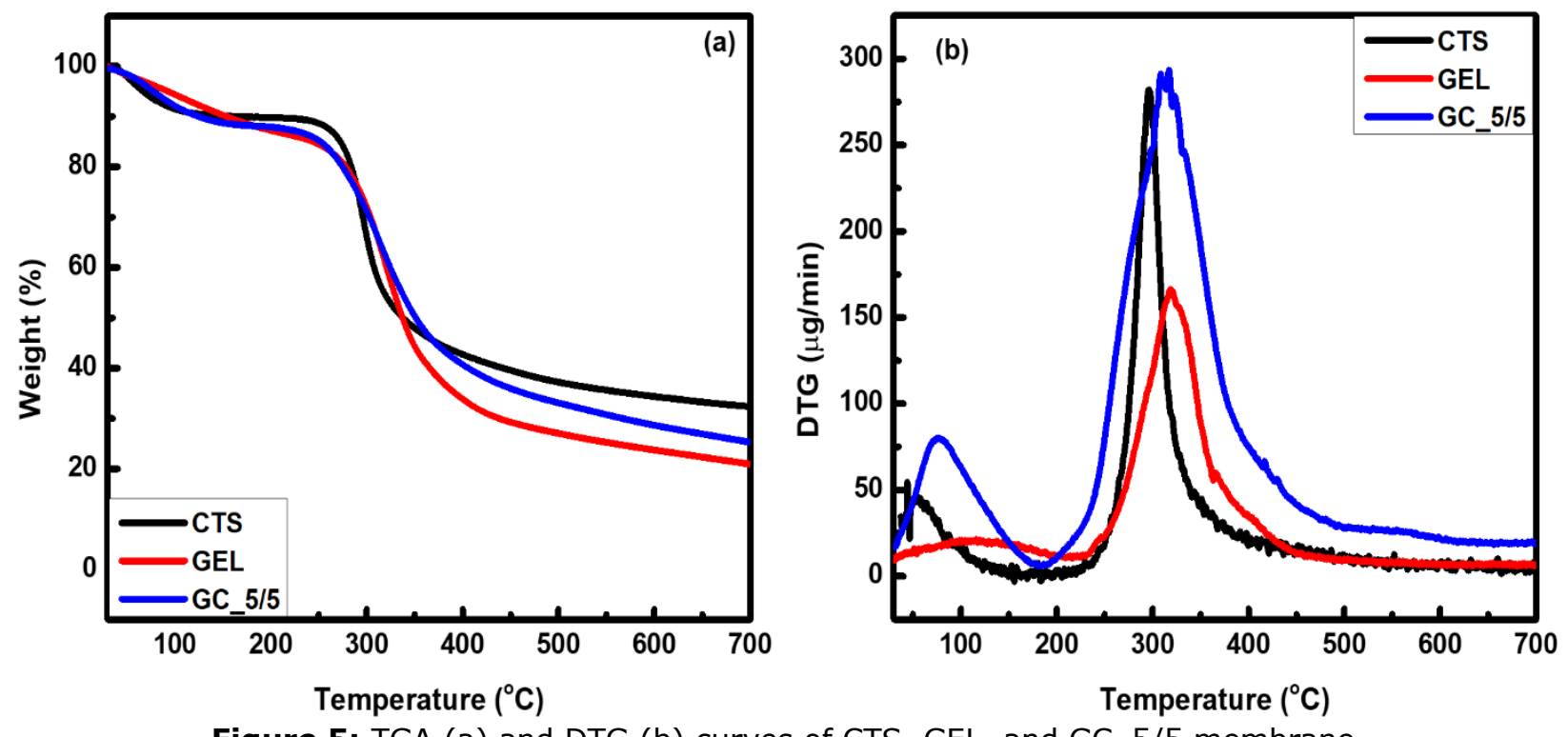

Figure 5: TGA (a) and DTG (b) curves of CTS, GEL, and GC_5/5 membrane.

\section{Mechanical Property of GC Membranes}

In addition to the above-suggested chemical, crystalline and thermal characterizations, a material's mechanical property is also seen as a rather important factor in this study. Actually, the hydrogel membranes reach lots of advantages, e.g., soft elastic property and promote the healed wound without any damage (17). Herein, the prepared GC membranes are used to directly investigate their mechanical property based on the values of stress at break and strain at break through the tensile test (Figure 6). It is obvious that the values of stress at break of the GC membranes are high with the decrease of weight ratio of GEL/CTS; it is the opposite for those of strain at break. Specifically, the GC_2/8 membrane has the highest stress and lowest strain values; on the other hand, those values are opposite for the GC_8/2 membrane. Additionally, the GC_5/5 membrane shows a higher value of stress at break comparing to the GC_6/4 and GC_8/2 membranes (i.e., the stress value range from GC_5/5 to GC_6/4 membranes: 0.26 $\mathrm{MPa}$; the stress value range from GC_5/5 to GC_6/4 membranes: $0.62 \mathrm{MPa}$ ); on the other hand, the stress value range of GC_5/5 membrane is lower little comparing to the GC_4/6 and GC_2/8 membranes (i.e., the stress value range from GC_5/5 to GC_4/6 membranes: $0.10 \mathrm{MPa}$; the stress value range from GC_5/5 to GC_2/8 membranes: $0.30 \mathrm{MPa}$ ). Furthermore, the GC_5/5 membrane shows a lower strain value compared to the GC_6/4 and GC_8/2 membranes (i.e., the strain value range from GC_5/5 to GC_6/4 membranes: $0.37 \%$; the strain value range from GC_5/5 to GC_6/4 membranes: $0.81 \%$ ), while the strain range of GC_5/5 membrane is higher little comparing to the GC_4/6 and GC_2/8 membranes (i.e., the strain value range from GC_5/5 to GC_4/6 membranes: $0.14 \%$; the strain value range from GC_5/5 to GC_2/8 membranes: $0.26 \%$ ). It indicates that the prepared GC membranes with various weight ratios of GEL/CTS are flexible and reach different stress and strain values. Besides, physical strength is seen as an important factor in biomedical applications. These show that the as-prepared GC membranes can be truly useful in the applications of biomedical fields, at same time that the GC_5/5 membrane can be selected as a promising membrane to apply in the fields of wound dressing or tissue-engineering applications. 


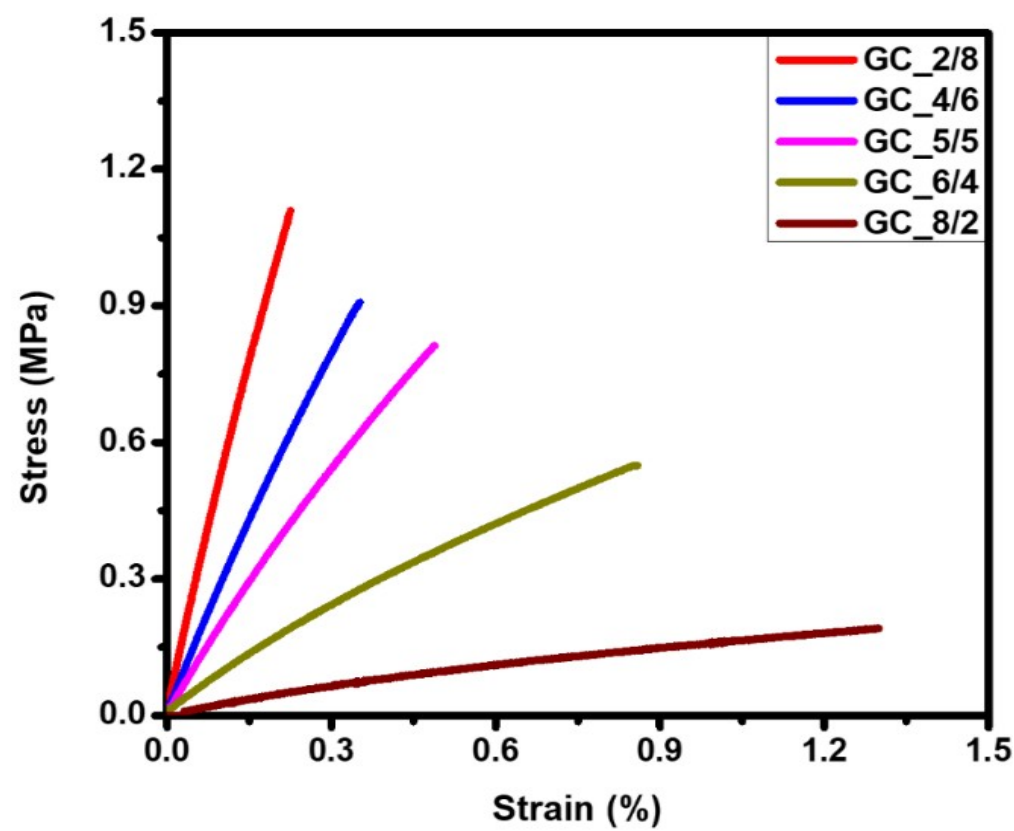

Figure 6: Stress-strain curves of the as-prepared GC membranes.

\section{Hydrophilic Property of GC Membranes}

As known, the hydrogel membranes reach lots of advantages, e.g., high water content, boost granulation, and epithelialization due to the moist environment (16). At the same time, the wettability and hydrophilic properties of a solid surface based on a liquid can be predicted from the use of contact angle. Herein, the hydrophilic property of the asprepared GC membrane surface is also investigated through the water contact angle method. Specifically, the picture of a water droplet on the

(a)

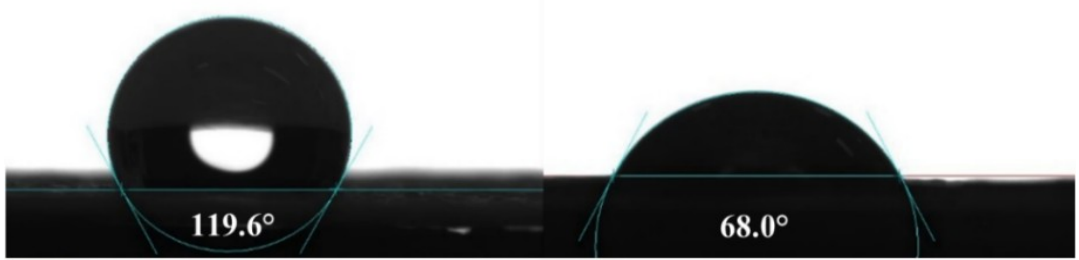

(c)

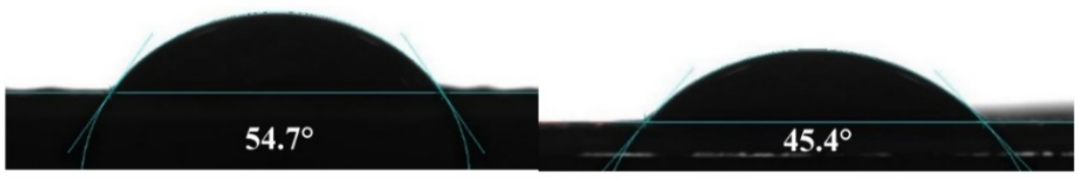

(e)

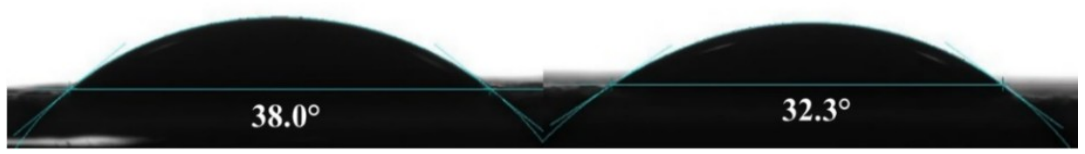

Figure 7: Contact angles of CTS (a), GC (b - f) membranes: GC_2/8; GC_4/6; GC_5/5; GC_6/4 and GC_8/2, respectively.
CTS surface is captured and shown in Figure 7a, resulting in that its water contact angle is obtained at $119.6^{\circ}$, as well as the hydrophobic nature of the CTS surface. On the other hand, the water contact angle of the as-prepared GC membrane surface significantly decreases with the increase of the GEL/CTS weight ratio (i.e., from $68.0^{\circ}$ to $32.3^{\circ}$ ) (Figure 7b-f) (38). These results further support the swelling test of the as-prepared GC membranes as well.

(b)

(d)

(f) 


\section{Swelling Property of GC Membranes}

Basically, the hydrogel membranes significantly swell in an aqueous medium that can be mainly due to their interacted networks, meaning they do not disintegrate in water at physiological $\mathrm{pH}$ and temperature (15). In addition to the abovementioned hydrophilic properties, the swelling property of the as-prepared GC membranes is conducted by immersing into distilled water at different times (i.e., 5 - $60 \mathrm{~min}$, r.t). For visual observation of the swelling studies (Figure 8), after
30 and 60 min immersing, GC $8 / 2$ and GC 6/4 membranes are almost soluble into the aqueous solution, in contrast for GC_5/5; GC_4/6, and GC 2/8 membranes. These show that the GC membranes can maintain stable swelling ability with a low weight ratio of GEL/CTS, which involves stronger intermolecular hydrogen bonds among the GC membranes. At the same time, these results are similar to the above-mentioned hydrophilic property of the membranes as well.
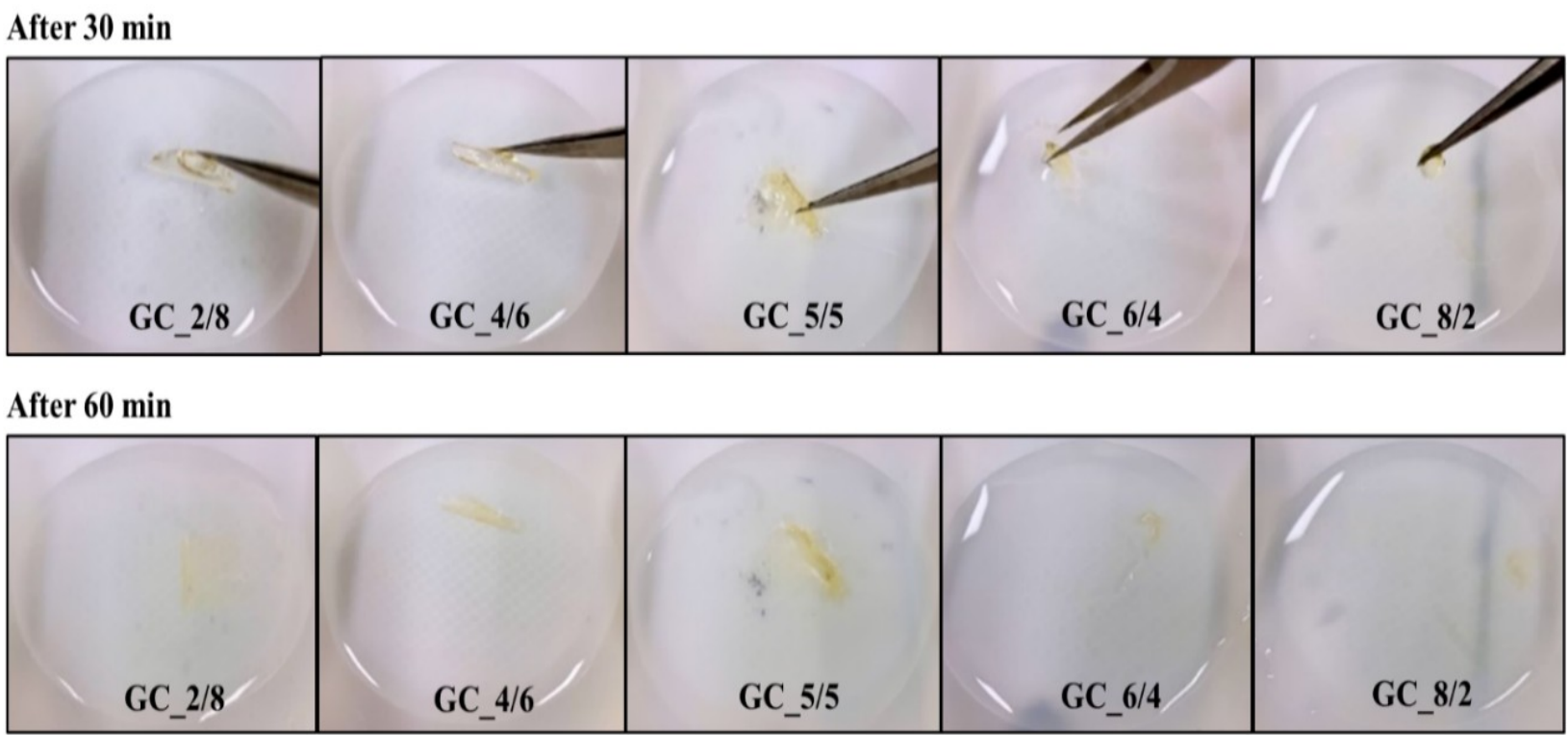

Figure 8: Swelling of all GC membranes after different immersing times.

Besides, each membrane's swelling rate at various immersing times is also concerned to be further investigated, as shown in Figure 9. Specifically, the swelling property of the GC membranes increases with the weight ratio of GEL/CTS within $5-60 \mathrm{~min}$ of immersing and sealing time period (i.e.: 51.6 $211.3 \%$ / GC_2/8 membrane; 87.7 - 248.3\% / GC_4/6 membrane; 107.3 - 270.4\% / GC_5/5 membrane; 148.2 - 390.9\% / GC 6/4 membrane; and 134.0 - 438.2\% / GC $8 / 2$ membrane). The main reason is that the addition of GEL can cause the hydrogel membrane structure to become looser, and the molecular chains in the system is more easily extended (39). Besides, the swelling rate range of each hydrogel membrane has significantly raised from 5 to 60 min of immersing and sealing time period (i.e.: $159.7 \%$ / GC_2/8 membrane; $160.6 \%$ / GC_4/6 membrane; $163.1 \%$ / GC_5/5 membrane; $242.7 \%$ / GC 6/4 membrane; and $304.2 \%$ / GC_8/2 membrane). The results suggest that the swelling property of each membrane increases rapidly from 5 to $60 \mathrm{~min}$. However, it slows down gradually after $45 \mathrm{~min}$, indicating that the swelling equilibrium of each membrane reaches at $45 \mathrm{~min}$. In general, the GC membranes manifest a good swelling behavior since the incorporation of GEL molecule, which is one of the common hydrophilic polymers. Concomitantly, the water- absorbing capacity of the hydrogel membranes is higher than their own weight. Additionally, the GC_5/5 membrane shows lower much swelling rate comparing to the GC_6/4 and GC_8/2 membranes (i.e.: the swelling rate range from GC_5/5 to GC_6/4 membranes: $41.0 \%$ / $5 \mathrm{~min}, 84.9 \%$ / 10 $\min , 105.9 \%$ / $30 \mathrm{~min}, 110.2 \%$ / $45 \mathrm{~min}$ and $120.5 \%$ / $60 \mathrm{~min}$; the swelling rate range from GC_5/5 to GC_8/2 membranes: $26.7 \%$ / 5 min, $120.2 \%$ / $10 \mathrm{~min}, 149.9 \%$ / $30 \mathrm{~min}, 69.8 \%$ / 45 $\min$ and $167.8 \%$ / $60 \mathrm{~min}$ ); on the other hand, the swelling rate range of $\mathrm{GC} 5 / 5$ membrane is higher little comparing to the GC_4/6 and GC $2 / 8$ membranes (i.e.: the swelling rate range from GC_5/5 to GC_4/6 membranes: $19.6 \%$ / 5 min, $22.7 \%$ / $10 \mathrm{~min}, 17.1 \%$ / $30 \mathrm{~min}, 22.3 \%$ / $45 \mathrm{~min}$ and $22.1 \%$ / $60 \mathrm{~min}$; the swelling rate range from GC_5/5 to GC_2/8 membranes: $55.7 \%$ / 5 min, $58.8 \%$ / $10 \mathrm{~min}, 40.1 \%$ / $30 \mathrm{~min}, 46.8 \%$ / $45 \mathrm{~min}$ and $59.2 \%$ / $60 \mathrm{~min}$ ). As a result, the GC membranes can maintain stable swelling ability with a low weight ratio of GEL/CTS, which involve to stronger intermolecular hydrogen bonds among the GC membranes. At the same time, the GC_5/5 membrane is chosen as a stable membrane to can apply for wound dressing or tissue-engineering applications. 


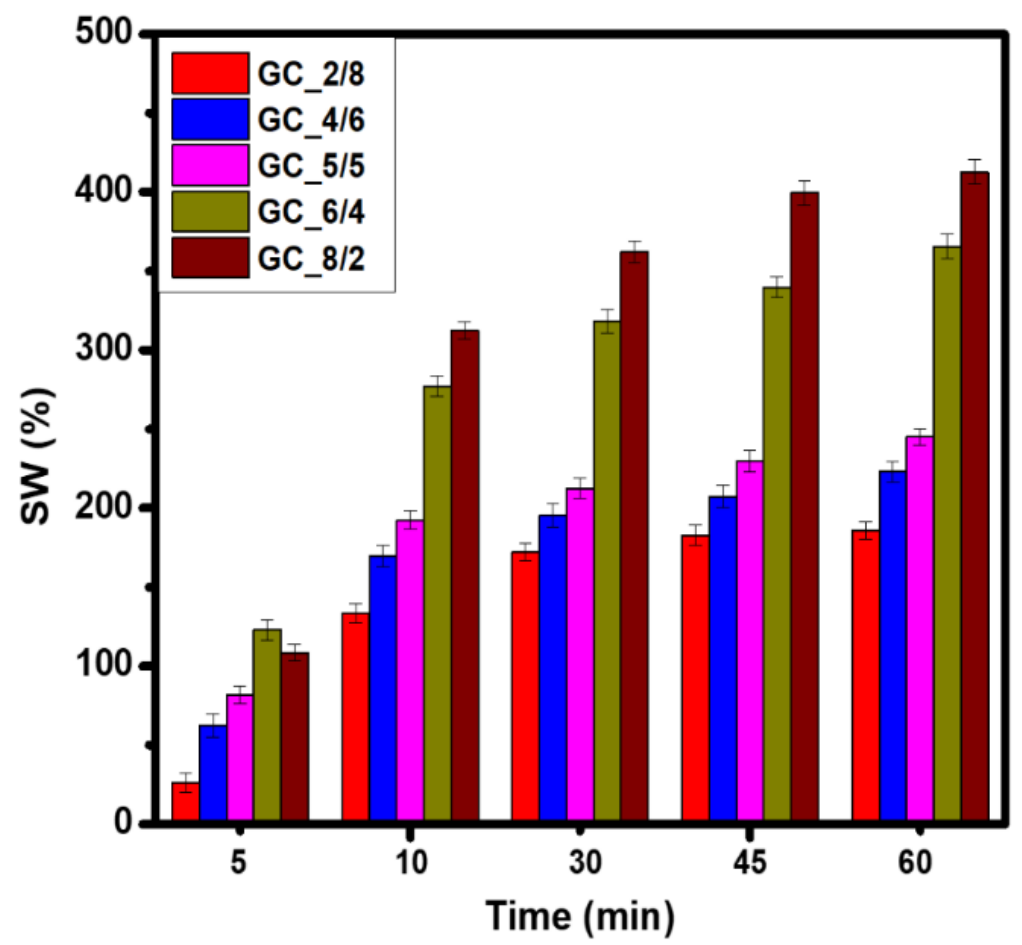

Figure 9: Swelling behavior of all GC membranes at room temperature.

\section{CONCLUSION}

In summary, GC membranes with various weight ratios of GEL/CTS have been successfully prepared by a simple in-situ method. The surface morphology of the as-prepared GC membranes, is found to be very smooth and homogeneous. For chemical and crystalline characterization of the as-prepared GC membranes, indicating that the incorporation of GEL in the membrane has decreased the crystallinity of CTS as well as appearing interaction between these molecules based on FT-IR and XRD studies. Besides, combining these natural polymer sources to form a hydrogel membrane is more stable in thermal property than pure materials. In addition to the above-mentioned characterizations, the hydrophilic property of GC membranes is investigated through contact angle measurements, resulting in their contact angle values significantly decrease with the increase of weight ratio of GEL/CTS (i.e., from $68.0^{\circ}$ to $32.3^{\circ}$ ). For the mechanical property of the GC membranes, the values of stress at break are high with the decrease of weight ratio of GEL/CTS, but it is the opposite for the values of strain at break. At the same time, the prepared GC membranes with various weight ratios of GEL/CTS are flexible and reach different stress and strain values. Moreover, the swelling equilibrium of each membrane has reached at $45 \mathrm{~min}$, as well as the GC membranes can maintain a stability in the swelling ability according to the low weight ratio of GEL/CTS, which involves to both the stronger intermolecular hydrogen bonds among the hydrogel membranes and the addition of GEL in the hydrogel membranes. These results suggest that the GC membrane can become a promising material (i.e., special for GC_5/5 membrane) in the field of wound dressing or tissue-engineering applications, at same time that the incorporation of GEL and CTS molecules is also considered as a nontoxic hydrogel membrane, owing to natural polymer sources.

\section{REFERENCES}

1. Vo TS, Vo TTBC, Suk JW, Kim K. Recycling performance of graphene oxide-chitosan hybrid hydrogels for removal of cationic and anionic dyes. Nano Convergence. 2020;7, 4:1-11. <DOI>.

2. Vo TS, Hossain MM, Jeong HM, Kim K. Heavy metal removal applications using adsorptive membranes. Nano Convergence. 2020;7, 36:2-26. $\leq \mathrm{DOI}$.

3. Vo TS, Vo TTBC. Preparation and Characterization of Bis-Propargyl-Succinate, and its Application in Preliminary Healing Ability of Crosslinked Polyurethane using "Azide-Alkyne" Click. Journal of Engineering Science and Technology Review. 2020;13(4): 110-6.

$\leq \mathrm{DOI}>$.

4. Vo TS, Vo TTBC, Tien TT, Singh NT. Enhancement of mechanical property of modified polyurethane with bis-butyl succinate. Journal of the Turkish Chemical Society Section A: Chemistry. 2021;8(2):519-26. DOI: <DOI>.

5. Vo TS, Vo TTBC. A Self-Healing Material Based on Microcapsules of Poly(Urea-Formaldehyde)/Bis- 
Propargyl-Succinate Containing in Polyurethane Matrix. Journal of the Turkish Chemical Society Section A: Chemistry. 2021;8(3):787-802. <DOI>

6. Velmurugan N, Kumar GG, Han SS, Nahm KS, Lee YS. Synthesis and Characterization of Potential Fungicidal Silver Nano-Sized Particles and Chitosan Membrane Containing Silver Particles. Iranian Polymer Journal. 2009;18(5 (107)):383-92.

7. Ti Feng J, Zhou J, Zhou J, Gao L, Xing Y, XuHui L. Synthesis and Characterization of Chitosan-based Schiff Base Compounds with Aromatic Substituent Groups. Iranian Polymer Journal. 2011;20(2):123-36.

8. KSV Krishna R, K Madhusudana R, Kumar PN, IlDoo C. Novel Chitosan-based pH Sensitive Micronetworks for the Controlled Release of 5Fluorouracil. Iranian Polymer Journal. 2010;19(4):265-76.

9. Gbenebor OP, Adeosun SO, Lawal GI, Jun S, Olaleye SA. Acetylation, Crystalline and Morphological Properties of Structural Polysaccharide from Shrimp Exoskeleton. Engineering Science and Technology, an International Journal. 2017;20(3):1155-65. <DOI>.

10. Peng $Z$, Li $Z$, Shen $Y$. Influence of Chemical Cross-Linking on Properties of Gelatin/Chitosan Microspheres. Polymer-Plastics Technology and Engineering. 2012;51(4):381-5. <DOI>.

11. Zamani A, Taherzadeh MJ. Effects of Partial Dehydration and Freezing Temperature on the Morphology and Water Binding Capacity of Carboxymethyl Chitosan-Based Superabsorbents. Industrial \& Engineering Chemistry Research. 2010;49(17):8094-9. <DOI>.

12. Adair A, Kaesaman A, Klinpituksa $P$. Superabsorbent materials derived from hydroxyethyl cellulose and bentonite: Preparation, characterization and swelling capacities. Polymer Testing. 2017;64:321-9. <DOI>.

13. Olad A, Pourkhiyabi M, Gharekhani H, Doustdar F. Semi-IPN superabsorbent nanocomposite based on sodium alginate and montmorillonite: Reaction parameters and swelling characteristics. Carbohydrate Polymers. 2018;190:295-306. $<$ DOI $>$.

14. Rop K, Mbui D, Njomo N, Karuku GN, Michira I, Ajayi RF. Biodegradable water hyacinth cellulosegraft-poly(ammonium acrylate-co-acrylic acid) polymer hydrogel for potential agricultural application. Heliyon. 2019;5(3):e01416. <DOI>.
15. Pal K, Banthia AK, Majumdar DK. Preparation and characterization of polyvinyl alcohol-gelatin hydrogel membranes for biomedical applications. AAPS PharmSciTech. 2007;8(1):E1-5. <DOI>.

16. Fan $L$, Yang $H$, Yang $J$, Peng $M$, $H u$ Preparation and characterization of chitosan/gelatin/PVA hydrogel for wound dressings. Carbohydrate Polymers. 2016;146:427-34. <DOI>.

17. Gonzalez JS, Maiolo AS, Hoppe CE, Alvarez VA. Composite Gels Based on Poly (Vinyl alcohol) for Biomedical Uses. Procedia Materials Science. 2012;1:483-90. <DOI>.

18. Dragan ES. Design and applications of interpenetrating polymer network hydrogels. A review. Chemical Engineering Journal. 2014;243:572-90. <DOI>.

19. Tokura S, Ueno K, Miyazaki S, Nishi N. Molecular Weight Dependent Antimicrobial Activity by Chitosan. In: New Macromolecular Architecture and Functions. Berlin, Heidelberg: Springer Berlin Heidelberg; 1996. p. 199-207. <DOI>.

20. Ceylan S, Alatepeli B. Evaluation of PVA/Chitosan Cryogels as Potential Tissue Engineering Scaffolds; Synthesis, cytotoxicity and genotoxicity investigations. Journal of the Turkish Chemical Society Section A: Chemistry. 2020;8(1):69-78. <DOI>.

21. Dwivedi A, Bharti P, Shukla S. Surface assimilation and corrosion inhibition characteristic of water soluble Polyvinyl Alcohol on mild steel surface in $0.5 \mathrm{M} \mathrm{HCl}$ solution. Journal of the Turkish Chemical Society Section A: Chemistry. 2021;8(1):217-28. <DOI>.

22. Okuyama K, Noguchi K, Miyazawa T, Yui T, Ogawa K. Molecular and Crystal Structure of Hydrated Chitosan. Macromolecules. 1997;30(19):5849-55. <DOI>.

23. Jayakumar R, Prabaharan M, Reis RL, Mano JF. Graft copolymerized chitosan-present status and applications. Carbohydrate Polymers. 2005;62(2):142-58. <DOl>.

24. Jayakumar R, Reis RL, Mano JF. Chemistry and Applications of Phosphorylated Chitin and Chitosan. E-Polymers. 2006;6(1):1-16. <DOI>.

25. Jayakumar R, Reis RL, Mano JF. Synthesis and Characterization of $\mathrm{N}$-methylenephenyl Phosphonic Chitosan. Journal of Macromolecular Science, Part A. 2007;44(3):271-5. <DOI>.

26. Kołodziejska I, Piotrowska B, Bulge M, Tylingo R. Effect of transglutaminase and 1-ethyl-3-(3dimethylaminopropyl) carbodiimide on the solubility 
of fish gelatin-chitosan films. Carbohydrate Polymers. 2006;65(4):404-9. <DOI>.

27. Sendemir-Urkmez A, Jamison RD. The addition of biphasic calcium phosphate to porous chitosan scaffolds enhances bone tissue developmentin vitro. Journal of Biomedical Materials Research Part A. 2007;81A(3):624-33. <DOI>.

28. Arvanitoyannis IS, Nakayama A, Aiba S. Chitosan and gelatin based edible films: state diagrams, mechanical and permeation properties. Carbohydrate Polymers. 1998;37(4):371-82. $\leq \mathrm{DOI}>$.

29. Achet $\mathrm{D}, \mathrm{He} X \mathrm{XW}$. Determination of the renaturation level in gelatin films. Polymer. 1995;36(4):787-91. <DOI>.

30. Fan M, Hu Q, Shen K. Preparation and structure of chitosan soluble in wide $\mathrm{pH}$ range. Carbohydrate Polymers. $2009 ; 78(1): 66-71$. $\leq \mathrm{DOI}$.

31. Lu S, Song X, Cao D, Chen Y, Yao K. Preparation of water-soluble chitosan. Journal of Applied Polymer Science. 2004;91(6):3497-503. <DOI>.

32. Qin C, Li H, XIAO Q, Liu Y, Zhu J, Du Y. Watersolubility of chitosan and its antimicrobial activity. Carbohydrate Polymers. 2006;63(3):367-74. $\leq \mathrm{DOI}$.

33. Borzacchiello A, Ambrosio L, Netti PA, Nicolais L, Peniche C, Gallardo A, et al. Chitosan-based hydrogels: Synthesis and characterization. Journal of Materials Science: Materials in Medicine. 2001;12(10):861-4. <DOI>.
34. Guan YL, Shao L, De Yao K. A study on correlation between water state and swelling kinetics of chitosan-based hydrogels. Journal of Applied Polymer Science. 1996;61(13):2325-35. <DOI .

35. Xiao C, Lu Y, Gao S, Zhang L. Characterization of konjac glucomannan-gelatin blend films. Journal of Applied Polymer Science. $2001 ; 79(9): 1596-602$. $\leq$ DOI $>$.

36. Cheng $M$, Deng J, Yang F, Gong Y, Zhao N, Zhang $X$. Study on physical properties and nerve cell affinity of composite films from chitosan and gelatin solutions. Biomaterials. 2003;24(17):287180. $\leq \mathrm{DOI}>$.

37. Zhai M, Zhao L, Yoshii F, Kume T. Study on antibacterial starch/chitosan blend film formed under the action of irradiation. Carbohydrate Polymers. 2004;57(1):83-8. <DOI>.

38. Delmar K, Bianco-Peled $\mathrm{H}$. The dramatic effect of small $\mathrm{pH}$ changes on the properties of chitosan hydrogels crosslinked with genipin. Carbohydrate Polymers. 2015;127:28-37. <DOI>.

39. Tomić SL, Mićić MM, Dobić SN, Filipović JM, Suljovrujić EH. Smart poly(2-hydroxyethyl methacrylate/itaconic acid) hydrogels for biomedical application. Radiation Physics and Chemistry. 2010;79(5):643-9. <DOI>. 
Vo et al. JOTCSA. 2021; 8(4): 1045-1056.

RESEARCH ARTICLE 